\title{
Improved Reproducibility of Protein Detection in Neuromelanin-containing Human Substantia Nigra Tissue enriched by Laser Microdissection
}

\section{Steffen Kösters}

Ruhr-Universitat Bochum Medizinische Fakultat https://orcid.org/0000-0001-6972-0653

Mandy Rettel

European Molecular Biology Laboratory

\section{Britta Eggers}

Ruhr-Universitat Bochum Medizinische Fakultat

\section{Peter Riederer}

Universitatsklinikum Wurzburg Klinik und Poliklinik fur Kinder- und Jugendpsychiatrie Psychosomatik und Psychotherapie

\section{Manfred Gerlach}

Universitatsklinikum Wurzburg Klinik und Poliklinik fur Kinder- und Jugendpsychiatrie Psychosomatik und Psychotherapie

\section{Mikhail Savitski}

European Molecular Biology Laboratory

Katrin Marcus ( $\nabla$ katrin.marcus@rub.de)

Ruhr-Universitat Bochum Medizinische Fakultat https://orcid.org/0000-0002-3313-8845

\section{Caroline May}

Ruhr-Universitat Bochum Medizinische Fakultat

\section{Methodology}

Keywords: substantia nigra, Neuromelanin, laser microdissection, sample preparation, in-solution digest, brain proteomics, SP3 protocol, trypsin digest, RapiGest ${ }^{\text {TM }}$

Posted Date: September 9th, 2020

DOI: https://doi.org/10.21203/rs.3.rs-70905/v1

License: (c) (i) This work is licensed under a Creative Commons Attribution 4.0 International License. Read Full License 


\section{Abstract}

Background: Processing of post-mortem human brain tissue samples like Neuromelanin-containing substantia nigra is a challenging task in proteomics research in the context of neurodegenerative diseases such as Parkinson's disease, due to the availability of only limited sample amounts. Therefore, a highly efficient lysis and digestion approach is needed to guarantee high reproducibility and a good quantification performance. We conducted a systematic study in order to compare qualitatively and quantitatively six different sample preparation protocols for substantia nigra brain tissue collected by laser microdissection.

Results: LC-MS/MS analysis revealed that the combination of formic acid and in-solution digestion with trypsin leads to an enhanced peptide and protein identification as well as a robust and reliable protein quantification with a very good reproducibility.

Conclusion: We recommend this protocol for future studies of human substantia nigra tissue as well as NM granules in the context of neurodegenerative disorders like Parkinson syndrome.

\section{Background}

Neuromelanin (NM) is a black, almost insoluble pigment that is present in the catecholaminergic, dopamine-producing neurons of the substantia nigra (SN) (1). It is located in the SN within the perikaryon (2). Together with proteins and lipids, it forms organelle-like granules surrounded by a double membrane $(3-5)$. During the aging process, the pigment in the form of NM granules increases in quantity and density (6). Whereas, people with Parkinson's disease (PD) show a pale brainstem due to a loss of NMcontaining neurons in the $\operatorname{SN}(7,8)$. On the one hand, it is assumed that the formation of NM granules protects the cell from accumulation of toxic and oxidative substances as well as from environmental toxins $(9,10)$. On the other hand, besides this neuroprotective function, there is evidence that NM granules can have neurotoxic effects $(11-13)$. Yet, the exact role of NM granules in the process of PD is not clearly understood.

Regardless of a neurological disorder, some proteomic studies of post-mortem SN tissue of healthy individuals lead to the assumption that due to a certain amount of lysosomal proteins NM granules are lysosomal related organelles or specialised autolysosomes that accumulate undegraded proteins (14, 15). Therefore, the analysis of $S N$ tissue as well as NM granules plays an important role for identifying, exploring and validating pathological processes (16-18). However, due to the limited availability and heterogeneity of human brain tissue, highly selective and reliable sampling methods are needed for further studies in the context of neurological disorders like PD.

For example, the use of laser microdissection (LMD) in combination with mass spectrometry (MS) is one of these methods. In general, LMD allows the selective analysis of specified human brain tissue areas, different cell types or single cells and even subcellular structures $(14,19-22)$. This selective isolation method offers several advantages for proteome analysis in the context of a neurological disorder. For 
example, the required $\mathrm{SN}$ tissue quantity can be significantly reduced compared to density gradient ultracentrifugation (14). At the same time, individual cells and subcellular structures can be cut from a tissue section semi-automatically. However, this process generates samples with low protein amounts, which can be a demanding task, because it may lead to low protein identification rates or results. Furthermore, the human brain is highly enriched in lipids $(23,24)$, which can cause some issues during the preparation and digestion steps. Therefore, this type of samples requires optimal sample preparation, which should ensure high reproducibility, efficiency and good proteome coverage.

To enable this and overcome the above described issues, we here introduce an optimized sample preparation protocol for low amounts of fresh frozen $\mathrm{SN}$ tissue containing neuromelanin granules enriched by LMD.

\section{Results}

We combined and compared different published protocols. Since the most common method for LMD samples is in-solution digestion with the serine protease trypsin, an adapted protocol was selected accordingly $(14,21)$. In addition, two published SP3 protocols based on paramagnetic beads were used, which are promisingly applicable for small sample volumes $(25,26)$. The protocols worked very well on cells like yeast, HeLa, mouse embryonic stem and neural progenitor cells $(25,26)$. For the lysis, besides RapiGest $^{\text {TI' }}$ and LDS as used in the mentioned protocols, formic acid was also tested as an alternative. This variant was successfully applied to muscle cells at our institute (27).

In total, six different combinations of tissue lysis and digestion were tested (see Table 1). For every protocol three different replicates were used. Each replicate contained one million $\mu \mathrm{m}^{2}$ post-mortem tissue from the same neurological healthy case (see Fig. 1).

For every replicate, the number of peptide spectrum matches (PSMs) unique peptides, identified and quantified protein groups (PGs) was determined. As an evaluation criterion the mean number as well as the standard deviation (SD) for every protocol was calculated. For the assessment of the analytical variability, three replicates of HeLa standards were added to the batch. Based on the numbers of quantified PGs an analytical variability of $6.8 \%$ (CV) was computed (see Table S2). 
Table 1

Overview of the used protocols with the corresponding numbers, names and references.

\begin{tabular}{|lll|}
\hline No. & Name & Ref. \\
\hline 1 & RapiGest $^{\mathrm{TM}}$ \& SP3 & $(25)$ \\
\hline 2 & LDS \& SP3 $^{\mathrm{TM}}$ \& modified SP3 & $(25)$ \\
\hline 3 & RapiGest $^{\mathrm{T}}$ (26) \\
\hline 4 & Formic acid \& modified SP3 $^{\mathrm{T}}$ \& in-solution digestion & $(26,27)$ \\
\hline 5 & RapiGest $^{\mathrm{M}}$ (21) & $(21,27)$ \\
\hline 6 & Formic acid \& in-solution digestion & $(25$ \\
\hline
\end{tabular}

The mean number of PSMs of each protocol is shown in Fig. 2A. The combination of formic acid with insolution digestion shows the highest number of PSMs (16685 PSMs). Protocol RapiGest ${ }^{\text {tM }}$ \& SP3 produced the lowest mean number of PSMs (8277 PSMs). These numbers result in the unique peptides shown in Fig. 2A. Again, formic acid \& in-solution digestion has the highest number of unique peptides (10313) and RapiGest ${ }^{\text {TM }} \&$ SP3 resulted in the lowest number of unique peptides (4780). Formic acid \& insolution digestion shows the lowest deviation in PSMs and unique peptides between the three replicates (black error bar, Fig. 2A).

In the next step, an evaluation of the identified and quantified PGs was performed. The results are shown in Fig. 2B. Formic acid \& in-solution digestion shows the highest number of identified (2123) and quantified (1596) PGs. The smallest number of PGs was achieved with formic acid \& modified SP3 (identified PGs: 1541 \& quantified PGs: 1137). Regarding the SD, formic acid \& in-solution digestion is again characterized by the smallest deviation for the identified and quantified PGs. In contrast, RapiGest ${ }^{\mathrm{TM}}$ \& SP3 protocol has the highest SD.

In summary, these results show that formic acid \& in-solution digestion quantitatively achieves optimal results at the level of peptides and proteins for small amounts of SN tissue enriched by LMD.

In order to confirm the previous results on a qualitative level, the ratio of the joint unique peptides to the total number of unique peptides between the three replicates was additionally determined for each protocol. This means a unique peptide that appears in only 3 of the 3 replicates meet this criterion. It was also performed for the identified and quantified PGs. The higher the calculated ratio in percentage, the higher the reproducibility for the results obtained from Fig. 2. Furthermore, the coefficient of variation (CV) was calculated for the quantified protein groups as an additional factor for the assessment of reproducibility and quantification. In this way, the CV can be used to make a statement about the performance of the quantification. A small CV indicates a low variance between the LFQ intensities of the three replicates. The calculated data are shown in Table 2 below. Formic acid \& in-solution digestion has 
the highest values at the calculated ratios and at the same time the lowest CV. In contrast, RapiGest ${ }^{\text {TM }}$ \& SP3, LDS \& SP3 and RapiGest ${ }^{\text {t" }}$ \& in-solution digestion have almost twice the CV and in some cases significantly lower ratios at both protein and peptide level.

Table 2

Assessment of reproducibility and quantification for the MaxQuant analysis without "match between runs". For each protocol, the number of consistently identified unique peptides, PGs and quantified PGs was established and divided by the corresponding total number. Consistently means that for example a unique peptide was detected in all three replicates. The calculated ratio is given in percentage. The CV for each quantified PG was determined. The given $\mathrm{CV}$ is the median percentage for all shared quantified PGs for each protocol. Protocols: RapiGest ${ }^{\text {TM }}$ \& SP3 (1), LDS \& SP3 (2), RapiGest ${ }^{\text {TM }}$ \& modified SP3 (3), Formic acid \& modified SP3 (4), RapiGest ${ }^{\text {TM }} \&$ in-solution digestion (5), Formic acid \& in-solution digestion (6). Abbreviations: Consistently (con.), identified (ident.), unique peptides (un. pep.), number (no.), quantified (quant.).

\begin{tabular}{|llllllll|}
\hline Protocol & $\begin{array}{l}\text { Con. ident. } \\
\text { un. pep. } \\
\text { /Total no. }\end{array}$ & $\begin{array}{l}\text { Total } \\
\text { no. un. } \\
\text { pep. }\end{array}$ & $\begin{array}{l}\text { Con. ident. } \\
\text { PGs /Total } \\
\text { no. }\end{array}$ & $\begin{array}{l}\text { Total } \\
\text { no. } \\
\text { ident. } \\
\text { PGS }\end{array}$ & $\begin{array}{l}\text { Con. quant. } \\
\text { PGs } / \text { Total } \\
\text { no. }\end{array}$ & $\begin{array}{l}\text { Total no. } \\
\text { quant. } \\
\text { PGs }\end{array}$ & $\begin{array}{l}\text { Median } \\
\text { CV } \\
\text { (Quant. } \\
\text { PGs) }\end{array}$ \\
\hline 1 & $33.0 \%$ & 7892 & $53.6 \%$ & 2094 & $28.1 \%$ & 2080 & $15.8 \%$ \\
\hline 2 & $31.3 \%$ & 9865 & $56.3 \%$ & 2240 & $28.0 \%$ & 2219 & $18.8 \%$ \\
\hline 3 & $38.2 \%$ & 9094 & $64.5 \%$ & 2102 & $36.0 \%$ & 2059 & $14.4 \%$ \\
\hline 4 & $39.6 \%$ & 8427 & $63.3 \%$ & 1764 & $36.6 \%$ & 1751 & $12.7 \%$ \\
\hline 5 & $37.1 \%$ & 9602 & $62.8 \%$ & 2404 & $30.2 \%$ & 2301 & $16.3 \%$ \\
\hline 6 & $52.8 \%$ & 14090 & $75.3 \%$ & 2390 & $47.6 \%$ & 2332 & $8.2 \%$ \\
\hline
\end{tabular}

These results give detailed information about different identified and quantified PGs between the replicates for every protocol. However, different lysis methods can generate different types of proteins, e.g. membrane proteins. In order to check whether there is a large discrepancy between formic acid \& insolution digestion and the other individual protocols, a comparison of the protein accession numbers was carried out. Only PGs identified in all three replicates were used. The associated Venn-diagrams are shown in Fig. 2. The lowest concordance is around 82\% (Fig. 2E, RapiGest ${ }^{\text {tw }}$ \& in-solution digestion). In comparison, formic acid \& in-solution digestion even shows a $99 \%$ match to the identified PGs of LDS \& SP3 (Fig. 2B). The average value for all five comparisons towards formic acid \& in-solution digestion is about $90 \%$. Therefore, this protocol has a very high concordance of PGs identified in all three replicates. Similar results can be observed for the PGs quantified in all three replicates (average value: $92 \%$; Figure S1). The average value of accordance for the PGs identified and quantified in one of the three replicates is lower but still above $80 \%$ (Figure S2 and Figure S3).

For a further literature comparison, we analyzed our data with the settings "match between runs". When using this setting we identified for formic acid \& in-solution digestion 11428 unique peptides and 2300 
PGs as well as quantified 1827 PGs (Figure A4). The results for all used protocols are shown in the supplementary information.

In summary, the combination of formic acid and in-solution digestion shows the best performance in terms of the number of resulting PSMs (Fig. 2A). It exhibits the most unique peptides (Fig. 2B), the highest number of identified PGs (Fig. 2C) as well as quantified PGs (Fig. 2D). Moreover, this protocol has the lowest SD (Fig. 2) and CV (see Table 2), but it equally has the highest percentage of consistently identified peptides, PGs and quantified PGs (see Table 2). Moreover, it has a very high level of consistency with the other protocols in terms of the identified and quantified PGs.

\section{Discussion}

The analysis of post-mortem human brain tissue samples especially from patients with a neurodegenerative disorder is a challenge in proteome research. Due to the limited availability of neurologically well-characterized patients, it is difficult to obtain enough samples that are balanced with respect to gender and age for a sufficiently large study. This is important in order to be able to make statistically validated statements that are not dependent on the results of a single patient. Another major challenge is the heterogeneity of the human brain. Neurological diseases lead to the degeneration of certain cell types, which in turn are located in certain areas of the brain or other subareas. The real challenge here is to selectively isolate these structures and thus enable a specific proteome study. Plum et al. showed the efficiency of a laser microdissection device and analysed the proteome of isolated NM granules in 2016 (14). This selective isolation or enrichment in turn leads to small sample material, which increases the risk of an unspecific and non-reproducible protein loss compared to larger sample quantities. In order to keep potential sample loss during this preparation as low as possible, a digestion approach is required that ensures high efficiency and good proteome coverage. On the other hand, a good reproducibility must be given in order to be able to draw an optimal comparison between different samples. A variant based on in-solution digestion has already been used for the processing of SN tissue and NM granules at our institute $(14,21)$. To improve the described requirements, various published protocols have been selected, such as a paramagnetic bead-based protocol that should be promising for small sample volumes (25). In 2017 a slightly modified version of this protocol was published (26). Both protocols have not been tested with fresh frozen human NM granules or SN tissue samples so far. In the described protocols, the lysis is performed by LDS or RapiGest ${ }^{\mathrm{T}}$. As an additional alternative, a variant with formic acid was chosen, as this protocol has already been successfully applied to muscle cells at our institute (27). In order to ensure optimal conditions for subsequent mass spectrometric analysis, a comparison of the various published lysis and digestion protocols was performed (see Table 1). The results show that the combination of formic acid lysis and in-solution digestion (protocol 6 ) has the highest number of peptide-spectrum matches, unique peptides, identified and quantified protein groups. At the same time, this protocol has the lowest standard deviation between the three replicates. On the other hand, the results of the other protocols partly show a very high SD especially the SP3 protocol (Fig. 2). In previous unpublished measurements at our institute we observed similar effects using SP3 protocol on even smaller sample amounts of human SN tissue enriched by laser microdissection. In 2019 
Davis et al. used a SP3 protocol for brain tissue of a cerebellar cortex enriched by laser microdissection (28). Between three replicates they did not measure a high SD as in our study. They used the same slides, though they used different lysis buffer. RapiGest ${ }^{\text {tw }}$, SDS or LDS have not been applied. This could mean that the combination of lysis buffer and SP3 protocol used here influences the sample preparation or rather the handling of the sample preparation.

Davis et al. also tested combinations of lysis buffers and digestion methods to maximize the number of identifications and quantitative performance for single-cell proteome studies (28). They analyzed 60000 $\mu \mathrm{m}^{2}$ of laser microdissected cerebellum tissue. With a combination of TFE buffer and SP3 digestion they identified 6399 peptides and 1521 proteins as well as quantified 958 proteins. For a comparison we analyzed our data also with the settings "match between runs" as Davis et al.

When using the setting "match between runs" we identified for formic acid \& in-solution digestion a higher number of unique peptides, identified and quantified PGs up to $190 \%$ (Figure A4). Since we collected more tissue area, higher numbers could be expected. The use of different lysis buffer may also provide reasons for these different numbers. However, another main difference is the collected brain tissue. We enriched especially SN tissue with NM granules, which are accumulations of NM, lipids and proteins. Therefore, it is important to find an optimal sample preparation for each tissue type.

Using the setting "match between runs", over 150 more PGs were quantified by MS/MS in comparison to analysis without "match between runs" (Figure A4). As expected this setting lead in general to an increase in identification numbers based on a comparison of signals with similar characteristics between the different mass spectrometric runs.

Besides these differences, it is worth mentioning that RapiGest ${ }^{\text {T"m }} \&$ in-solution digestion has a much smaller difference between the mean number of unique peptides and the mean number of PGs compared to formic acid \& in-solution digestion. This implies that formic acid \& in-solution digestion has a higher sequence coverage, because on average more unique peptides were detected for each protein. The only difference between the two protocols is the lysis with RapiGest ${ }^{\mathrm{TM}}$ or formic acid. Thus, these results indicate a better lysis or rather denaturation of the samples by formic acid, whereby a more efficient digestion can be achieved. Consequently, these results indicate a good qualitative evaluation after application of formic acid \& in-solution digestion, since an increased number of peptides per protein can lead to an improved quantification. The quality of the quantification results is particularly important for a differential study. Since only mass spectrometry-based quantitative proteomics can provide insights into the function and dynamics of biological systems (29). Therefore, the coefficient of variation (CV) for the quantified PGs between the three replicates was additionally determined for each protocol (see Table 2). In this way, a more precise statement about the quality of the quantification could be made. The combination of formic acid and in-solution digestion showed the lowest CV. Thus, the variance of the intensities between the measured replicates is the smallest. This in turn speaks for a robust and reliable protein quantification as well as for a very good reproducibility. However, if the protein coverage is not very high and it shows huge differences to other sample preparation protocols, a differential proteome 
study would not reflect all biochemical processes. Therefore, we compared the identified and quantified PGs from formic acid \& in-solution digestion with the other protocols. The results show a very high accordance for this protocol.

\section{Conclusion}

Our systematic evaluation of different sample preparation protocols exhibited a combination of formic acid and in-solution digestion, being best suited for fresh frozen SN tissue samples. Indeed, different samples will behave differently, and for every sample type a systematic evaluation to find the best sample preparation protocol is recommended. It is important to check, which protocol fits best regarding reproducibility, efficiency and a good proteome coverage. The evaluation of quantification must not only be based on the pure number of quantified proteins but should also include assessments such as the CV or the calculation of the consistently quantified PGs relative to the total number as shown. Based on the presented data, the combination of formic acid and in-solution digestion with trypsin leads to an enhanced peptide and protein identification as well as a robust and reliable protein quantification. Besides that, the protocol shows a very good reproducibility and the highest quantification performance. Hence, we recommend this protocol for future studies of human $\mathrm{SN}$ tissue as well as NM granules in the context of neurodegenerative disorders like Parkinson's syndrome. In addition, the protocol can also be applied to other tissues such as muscle tissue.

\section{Methods}

The post-mortem human brain tissue was provided by the University of Würzburg. All subjects died from natural death, their brains were dissected and stored directly at $80^{\circ} \mathrm{C}$. Cryosectioning, staining and enrichment of fresh frozen brain tissue was performed as described in Molina et al (21). For each sample one million $\mu \mathrm{m}^{2}$ tissue was collected via LMD (PALM MicroBeam, Carl Zeiss Microscopy GmbH, Jena, Germany).

For tissue lysis three different approaches were used (a-c, see below); for each approach, three enriched tissue samples were first pooled and then split into three technical replicates. Before splitting the tissue, lysis was performed as following:

1. Tissue lysis with RapiGest ${ }^{\mathrm{Tm}}: 7 \mu \mathrm{L}$ of $1 \%$ RapiGest $^{\mathrm{TM}}$ solution was added to every sample. Reaction tubes were sonicated and cooled down in ice water for 30 seconds. This procedure was repeated four times to extract proteins from the tissue.

2. Tissue lysis with formic acid was performed with slight modifications previously described in 2013 (27): LMD tissue samples were dried in a SpeedVac ${ }^{\mathrm{TM}}$ (Concentrator plus, Eppendorf $\mathrm{GmbH}$, Hamburg, Germany) to remove solvent. $40 \mu \mathrm{L}$ of formic acid was added to each reaction tube. After 20 minutes incubation time, samples were shortly centrifuged, sonicated for 10 minutes and again shortly centrifuged. After being combined and splitted, samples were dried in a SpeedVac ${ }^{\mathrm{TM}}$, and $50 \mu \mathrm{L}$ of $50 \mathrm{mM}$ ammonium bicarbonate solution was added to each sample. 
3. Tissue lysis with LDS: $12.5 \mu \mathrm{L}$ of $4 x$ LDS buffer was added to every tissue sample. Reaction tubes were incubated at $95^{\circ} \mathrm{C}$ for 5 minutes.

After extraction, proteins were digested with trypsin. Three different digestion protocols were used. The applied combinations of tissue lysis and digestion protocols are shown in Table 1. For the single-pot, solid phase-enhanced sample-preparation (SP3) the protocol was used published in 2014 (25). For the modified single-pot, solid phase-enhanced sample-preparation (modified SP3) the protocol was used described in 2017 (26). The in-solution digestion was performed with slight modifications as described in Molina et al (21). For the latter protocol, samples were first reduced with $1 \mu \mathrm{L} 250 \mathrm{mM}$ DTT for 30 minutes at $60{ }^{\circ} \mathrm{C}$ and then alkylated with $1.4 \mu \mathrm{L} 0.55 \mathrm{M}$ iodoacetamide for 30 minutes in the dark at room temperature. Afterwards, the samples were heated up to $95^{\circ} \mathrm{C}$ for 5 minutes. Proteins were digested with $7 \mu \mathrm{L}$ trypsin-solution ( $13.3 \mathrm{ng} / \mu \mathrm{L}$ trypsin in $37.5 \mu \mathrm{L} 50 \mathrm{mM}$ ammonium bicarbonate) for 4 hours at $37^{\circ} \mathrm{C}$. The reaction was stopped by adding $3.25 \mu \mathrm{L} \mathrm{10 \%} \mathrm{trifluoroacetic} \mathrm{acid} \mathrm{to} \mathrm{each} \mathrm{sample} \mathrm{and} \mathrm{incubating}$ them for another 30 minutes at $37^{\circ} \mathrm{C}$. After centrifugation for 15 minutes at $4{ }^{\circ} \mathrm{C}$ and $10000 \mathrm{rpm}$, the supernatant of each sample was transferred into a new glass vial, dried in a SpeedVac ${ }^{\mathrm{TM}}$ and absorbed in $24 \mu \mathrm{L} 0.1 \%$ TFA solution.

LC-MS/MS was performed with a hybrid quadrupole-orbitrap mass spectrometer (Q Exactive HF, Thermo Fisher Scientific Inc., Bremen), coupled with a nano HPLC system (UltiMate 3000 RSLC, Dionex, Idstein, Germany) using the following solvent buffer system: (A) $0.1 \%$ formic acid; (B) $84 \%$ acetonitrile, $0.1 \%$ formic acid. $8 \mu \mathrm{L}$ of every sample was used and combined with $8 \mu \mathrm{L}$ of $0.1 \%$ TFA solution for the following analysis. $15 \mu \mathrm{L}$ per sample was injected onto a C-18 trap column (Thermo Fisher Scientific, Germany, $100 \mu \mathrm{m} \times 2 \mathrm{~cm}$, particle size $5 \mu \mathrm{m}$, pore size $100 \AA$, C18) with $0.1 \%$ trifluoroacetic acid and a flow rate of $30 \mu \mathrm{L} /$ minute. Peptides were transferred onto the analytical C18 column (Thermo Fisher Scientific, Germany, $75 \mu \mathrm{m} \times 50 \mathrm{~cm}$, particle size $2 \mu \mathrm{m}$, pore size $100 \AA)$, and were separated with a flow rate of $400 \mathrm{nl} /$ minute using a solvent gradient of $5 \%$ to $35 \% \mathrm{~B}$ for 98 minutes. Washing of the column was performed for 7 minutes with $95 \%$ B, and was then returned to $5 \%$ B. After each measured sample, equilibration of the column was done via a 50 minute washing step. The HPLC system was connected online to the mass spectrometer via a nano-electrospray ion source. The scan range was defined as $350-$ $1,400 \mathrm{~m} / \mathrm{z}$ with a resolution of 60,000 for the detection of precursor ions (AGC target 3e6, maximum injection time $80 \mathrm{~ms}$ ). Fragment ions were generated from the top ten most abundant precursor ions per cycle by using higher energy collisioninduced dissociation (HCD) at a normalized collision energy (NCE) of $27 \%$. MS/MS scans were performed in a $1.6 \mathrm{~m} / \mathrm{z}$ wide isolation window with a resolution of 30,000 , whereby the fixed first mass was set to $100 \mathrm{~m} / \mathrm{z}$ (AGC 1e6, maximum injection time $120 \mathrm{~ms}$ ).

All samples were measured in random order. For the assessment of the analytical variability, three replicates of HeLa standards were added to the batch. The MS data files were evaluated with the free software MaxQuant (version 1.6.0.16). Data was matched with a UniProt database (UniProtKB/SwissProt UniProt release 2018_04; downloaded 2018-05-09). Trypsin was specified as used enzyme and a maximum of two missed cleavages was selected. Only unique peptides were used for protein quantification. Carbamidomethylation (C) was selected as a fixed modification. Oxidation (M) was 
selected as variable modification. Further variable modifications were selected for each protocol differently. Deamidation (NQ) was selected for protocol 1-4 and 6. Whereas Carbamidomethylation and Amidine were selected for protocol 5 and 6 . All peptides with selected modifications were added and used for quantification. Further search parameters have been taken from the settings of MaxQuant such as $0.01 \mathrm{FDR}$ and MS/MS tolerance (FTMS: 20 ppm, ITMS: $0.5 \mathrm{Da}$ ).

\section{List Of Abbreviations}

CV (coefficient of variation); LDS (lithium dodecyl sulphate); LMD (laser microdissection); NM (Neuromelanin); PD (Parkinson's disease); PEN (polyethylene naphthalate); PG (protein group); PSM (peptide spectrum match); SD (standard deviation); SDS (sodium dodecyl sulphate); SN (substantia nigra); SP3 (Single-Pot Solid Phase-enhanced sample preparation)

\section{Declarations}

\section{Ethics approval and consent to participate}

The use of post-mortem human brain tissue was approved and consented by the Ethics Committee of the Ruhr-University Bochum (4760-13).

\section{Consent for publication}

Not applicable.

\section{Availability of data and materials}

The datasets generated and/or analysed during the current study are available in the ProteomeXchange Consortium via the Pride (30) partner repository with the dataset idenfier PXD015413.

\section{Competing interests}

The authors declare that they have no competing interest.

\section{Funding}

This work was supported by the Deutsche Parkinson Gesellschaft, Medical Faculty at RUB (FoRUM), the German Federal Ministry of Education and Research (WTZ with Brazil, FKZ 01DN14023), the HUPO Brain Proteome Project (HBPP) and PURE, a project of North-Rhine Westphalia, a federal German state. No funding bodies had any role in study design, data collection and analysis, decision to publish, or preparation of the manuscript. No specific funding was received for this study.

\section{Authors' contributions}


Conceptualization: Steffen Kösters, Caroline May

Data curation: Steffen Kösters

Funding acquisition: Katrin Marcus, Caroline May

Investigation: Steffen Kösters, Mandy Rettel

Methodology: Steffen Kösters, Mandy Rettel, Britta Eggers, Mikhail Savitski, Caroline May

Project administration: Katrin Marcus, Caroline May, Mikhail Savitski

Resources: Peter Riederer, Manfred Gerlach, Katrin Marcus, Mikhail Savitski

Software: Katrin Marcus, Mikhail Savitski

Validation: Steffen Kösters, Caroline May

Writing - original draft: Steffen Kösters,

Writing - reviewing \& editing: Peter Riederer, Manfred Gerlach, Mandy Rettel, Mikhail Savitski, Steffen Kösters, Katrin Marcus, Caroline May

\section{Acknowledgements}

Not applicable.

\section{References}

1. Bogerts B. A brainstem atlas of catecholaminergic neurons in man, using melanin as a natural marker. J Comp Neurol. 1981;197(1):63-80.

2. Duffy PE, Tennyson VM. Phase and Electron Microscopic Observations of Lewy Bodies and Melanin Granules in Substantia Nigra and Locus Caeruleus in Parkinsons Disease. Journal of Neuropathology and Experimental Neurology. 1965;24(3):398-\&.

3. Engelen M, Vanna R, Bellei C, Zucca FA, Wakamatsu K, Monzani E, et al. Neuromelanins of human brain have soluble and insoluble components with dolichols attached to the melanic structure. PLoS One. 2012;7(11):e48490.

4. Sulzer D, Mosharov E, Talloczy Z, Zucca FA, Simon JD, Zecca L. Neuronal pigmented autophagic vacuoles: lipofuscin, neuromelanin, and ceroid as macroautophagic responses during aging and disease. Journal of Neurochemistry. 2008;106(1):24-36.

5. Zecca L, Costi P, Mecacci C, Ito S, Terreni M, Sonnino S. Interaction of human substantia nigra neuromelanin with lipids and peptides. Journal of Neurochemistry. 2000;74(4):1758-65. 
6. Halliday GM, Fedorow H, Rickert CH, Gerlach M, Riederer P, Double KL. Evidence for specific phases in the development of human neuromelanin. Journal of Neural Transmission. 2006;113(6):721-8.

7. Dickson DW, Braak H, Duda JE, Duyckaerts C, Gasser T, Halliday GM, et al. Neuropathological assessment of Parkinson's disease: refining the diagnostic criteria. Lancet Neurol. 2009;8(12):11507.

8. Lees AJ, Selikhova M, Andrade LA, Duyckaerts C. The black stuff and Konstantin Nikolaevich Tretiakoff. Movement Disorders. 2008;23(6):777-83.

9. Paris I, Lozano J, Perez-Pastene C, Munoz P, Segura-Aguilar J. Molecular and Neurochemical Mechanisms in PD Pathogenesis. Neurotoxicity Research. 2009;16(3):271-9.

10. Zecca L, Zucca FA, Wilms H, Sulzer D. Neuromelanin of the substantia nigra: a neuronal black hole with protective and toxic characteristics. Trends in Neurosciences. 2003;26(11):578-80.

11. Zareba M, Bober A, Korytowski W, Zecca L, Sarna T. The Effect of a Synthetic Neuromelanin on Yield of Free Hydroxyl Radicals Generated in Model Systems. Biochimica Et Biophysica Acta-Molecular Basis of Disease. 1995;1271(2-3):343-8.

12. Karlsson O, Lindquist NG. Melanin affınity and its possible role in neurodegeneration. J Neural Transm (Vienna). 2013;120(12):1623-30.

13. Zucca FA, Segura-Aguilar J, Ferrari E, Munoz P, Paris I, Sulzer D, et al. Interactions of iron, dopamine and neuromelanin pathways in brain aging and Parkinson's disease. Prog Neurobiol. 2017;155:96119.

14. Plum S, Steinbach S, Attems J, Keers S, Riederer P, Gerlach M, et al. Proteomic characterization of neuromelanin granules isolated from human substantia nigra by laser-microdissection. Sci Rep. 2016;6:37139.

15. Zucca FA, Vanna R, Cupaioli FA, Bellei C, De Palma A, Di Silvestre D, et al. Neuromelanin organelles are specialized autolysosomes that accumulate undegraded proteins and lipids in aging human brain and are likely involved in Parkinson's disease. NPJ Parkinsons Dis. 2018;4:17.

16. Schonberger SJ, Edgar PF, Kydd R, Faull RL, Cooper GJ. Proteomic analysis of the brain in Alzheimer's disease: molecular phenotype of a complex disease process. Proteomics. 2001;1(12):1519-28.

17. Ping L, Duong DM, Yin L, Gearing M, Lah JJ, Levey Al, et al. Global quantitative analysis of the human brain proteome in Alzheimer's and Parkinson's Disease. Sci Data. 2018;5:180036.

18. Li KW, Ganz AB, Smit AB. Proteomics of neurodegenerative diseases: analysis of human postmortem brain. J Neurochem. 2018.

19. Kley RA, Maerkens A, Leber Y, Theis V, Schreiner A, van der Ven PF, et al. A combined laser microdissection and mass spectrometry approach reveals new disease relevant proteins accumulating in aggregates of filaminopathy patients. Mol Cell Proteomics. 2013;12(1):215-27.

20. Guttsches AK, Brady S, Krause K, Maerkens A, Uszkoreit J, Eisenacher M, et al. Proteomics of rimmed vacuoles define new risk allele in inclusion body myositis. Ann Neurol. 2017;81(2):227-39. 
21. Molina M, Steinbach S, Park YM, Yun SY, Di Lorenzo Alho AT, Heinsen H, et al. Enrichment of single neurons and defined brain regions from human brain tissue samples for subsequent proteome analysis. Journal of neural transmission (Vienna, Austria : 1996). 2015;122(7):993-1005.

22. Plum S, Steinbach S, Abel L, Marcus K, Helling S, May C. Proteomics in neurodegenerative diseases: Methods for obtaining a closer look at the neuronal proteome. Proteomics Clin Appl. 2014.

23. O'Brien JS, Sampson EL. Lipid composition of the normal human brain: gray matter, white matter, and myelin. J Lipid Res. 1965;6(4):537-44.

24. Cermenati G, Mitro N, Audano M, Melcangi RC, Crestani M, De Fabiani E, et al. Lipids in the nervous system: from biochemistry and molecular biology to patho-physiology. Biochim Biophys Acta. 2015;1851(1):51-60.

25. Hughes CS, Foehr S, Garfield DA, Furlong EE, Steinmetz LM, Krijgsveld J. Ultrasensitive proteome analysis using paramagnetic bead technology. Mol Syst Biol. 2014;10:757.

26. Sielaff M, Kuharev J, Bohn T, Hahlbrock J, Bopp T, Tenzer S, et al. Evaluation of FASP, SP3, and iST Protocols for Proteomic Sample Preparation in the Low Microgram Range. J Proteome Res. 2017;16(11):4060-72.

27. Maerkens A, Kley RA, Olive M, Theis V, van der Ven PF, Reimann J, et al. Differential proteomic analysis of abnormal intramyoplasmic aggregates in desminopathy. J Proteomics. 2013;90:14-27.

28. Davis S, Scott C, Ansorge O, Fischer R. Development of a Sensitive, Scalable Method for Spatial, CellType-Resolved Proteomics of the Human Brain. J Proteome Res. 2019;18(4):1787-95.

29. Ankney JA, Muneer A, Chen X. Relative and Absolute Quantitation in Mass Spectrometry-Based Proteomics. Annu Rev Anal Chem (Palo Alto Calif). 2018;11(1):49-77.

30. Perez-Riverol Y, Csordas A, Bai J, Bernal-Llinares M, Hewapathirana S, Kundu DJ, et al. The PRIDE database and related tools and resources in 2019: improving support for quantification data. Nucleic Acids Res. 2019;47(D1):D442-d50.

\section{Figures}




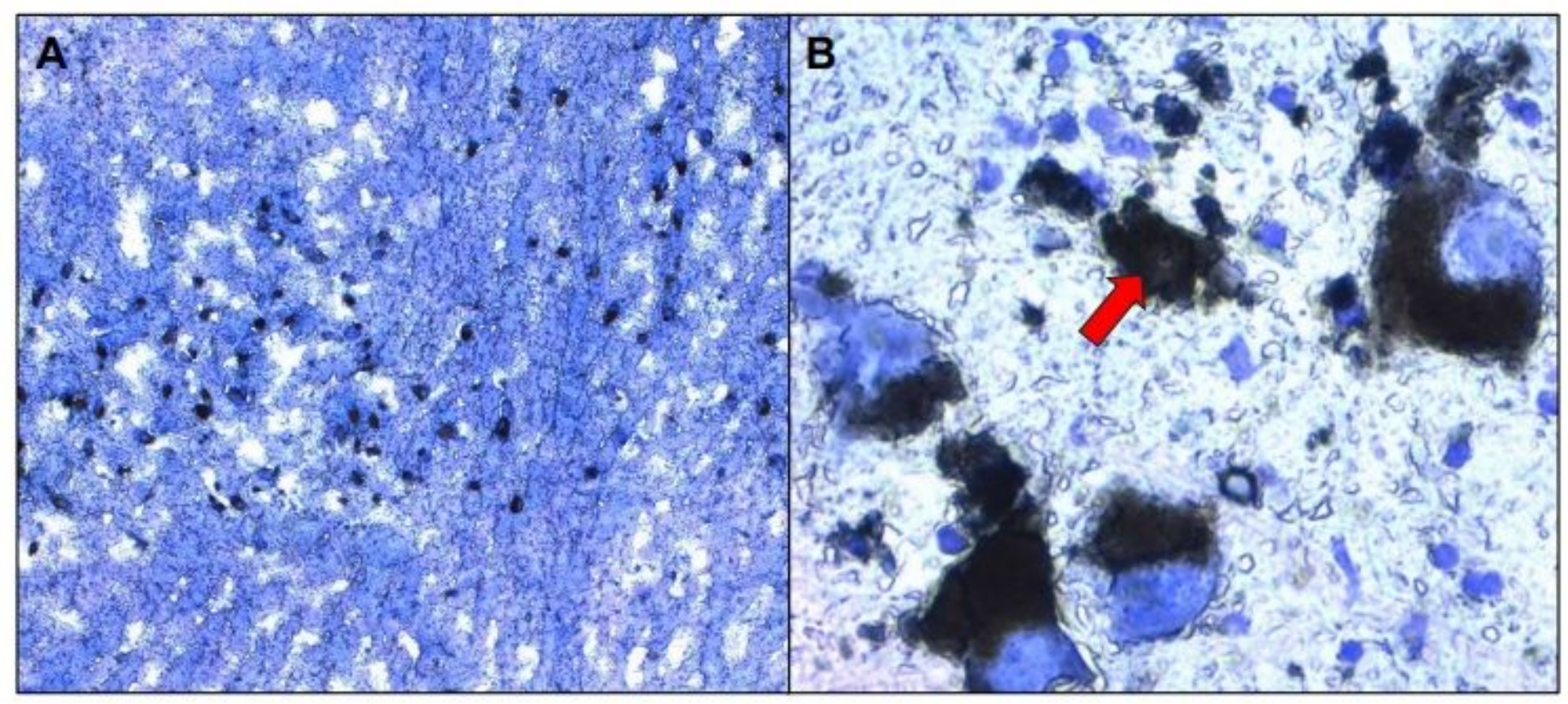

Figure 1

LMD images of post-mortem human substantia nigra tissue stained with cresyl violet. (A) Human substantia nigra pars compacta tissue at a magnification of 50x. (B) Human substantia nigra pars compacta tissue at a magnification of 400x. Red arrow: neuromelanin granules example. 


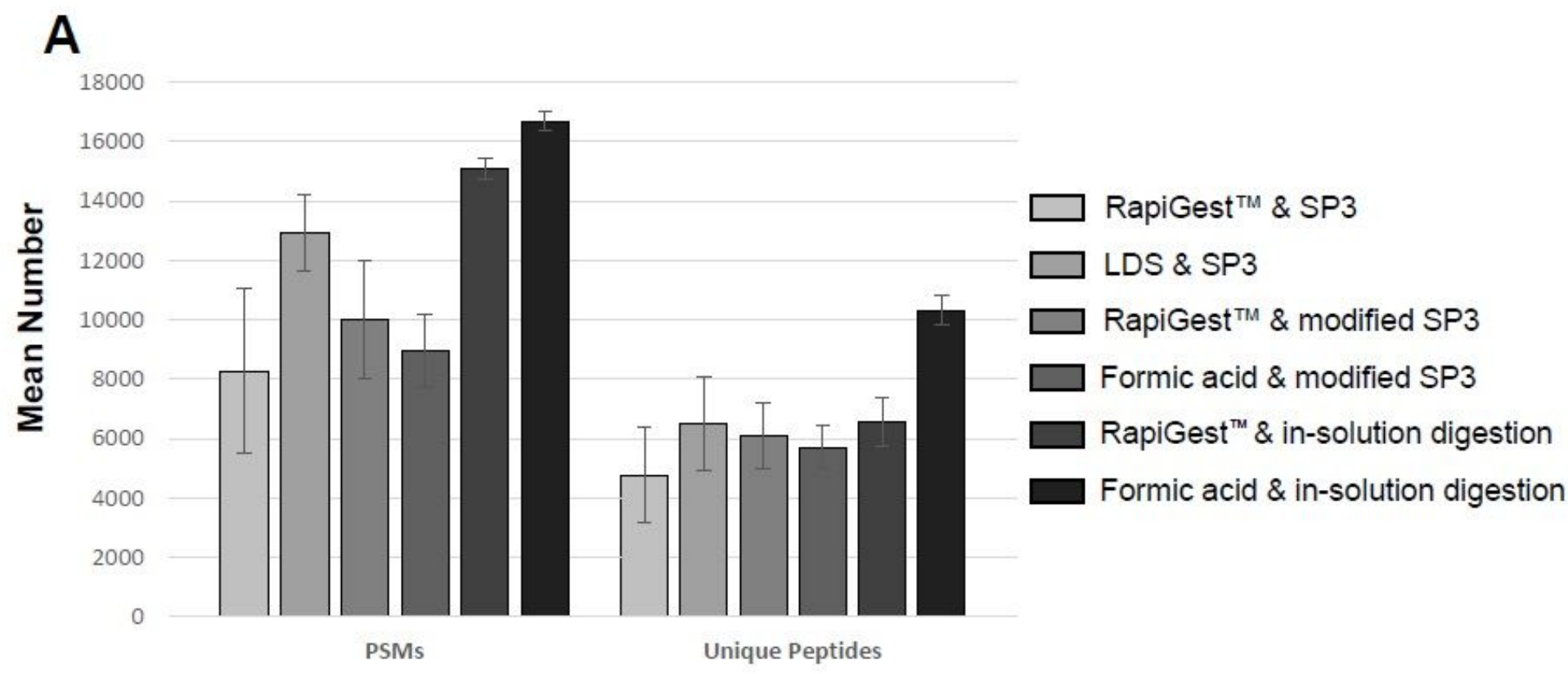

B

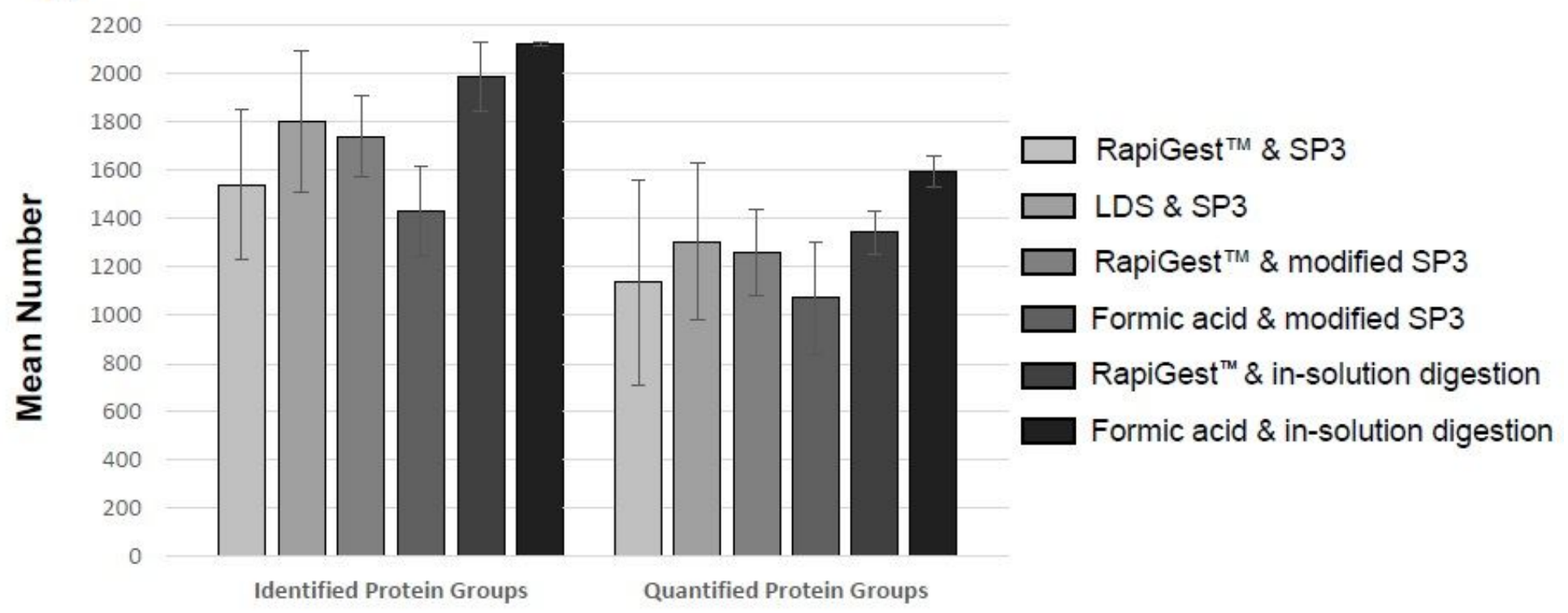

Figure 2

Mean number of PSMs (A), unique peptides (A), identified PGs (B) and quantified PGs (B). The error bars provide information about the SD between the three replicates. Protocol 1: RapiGest ${ }^{\mathrm{Tm}}$ \& SP3; Protocol 2: LDS \& SP3; Protocol 3: RapiGest ${ }^{\text {TM }}$ \& modified SP3, Protocol 4: Formic acid \& modified SP3; Protocol 5: RapiGest $^{\text {tM }} \&$ in-solution digestion; Protocol 6: Formic acid \& in-solution digestion. 

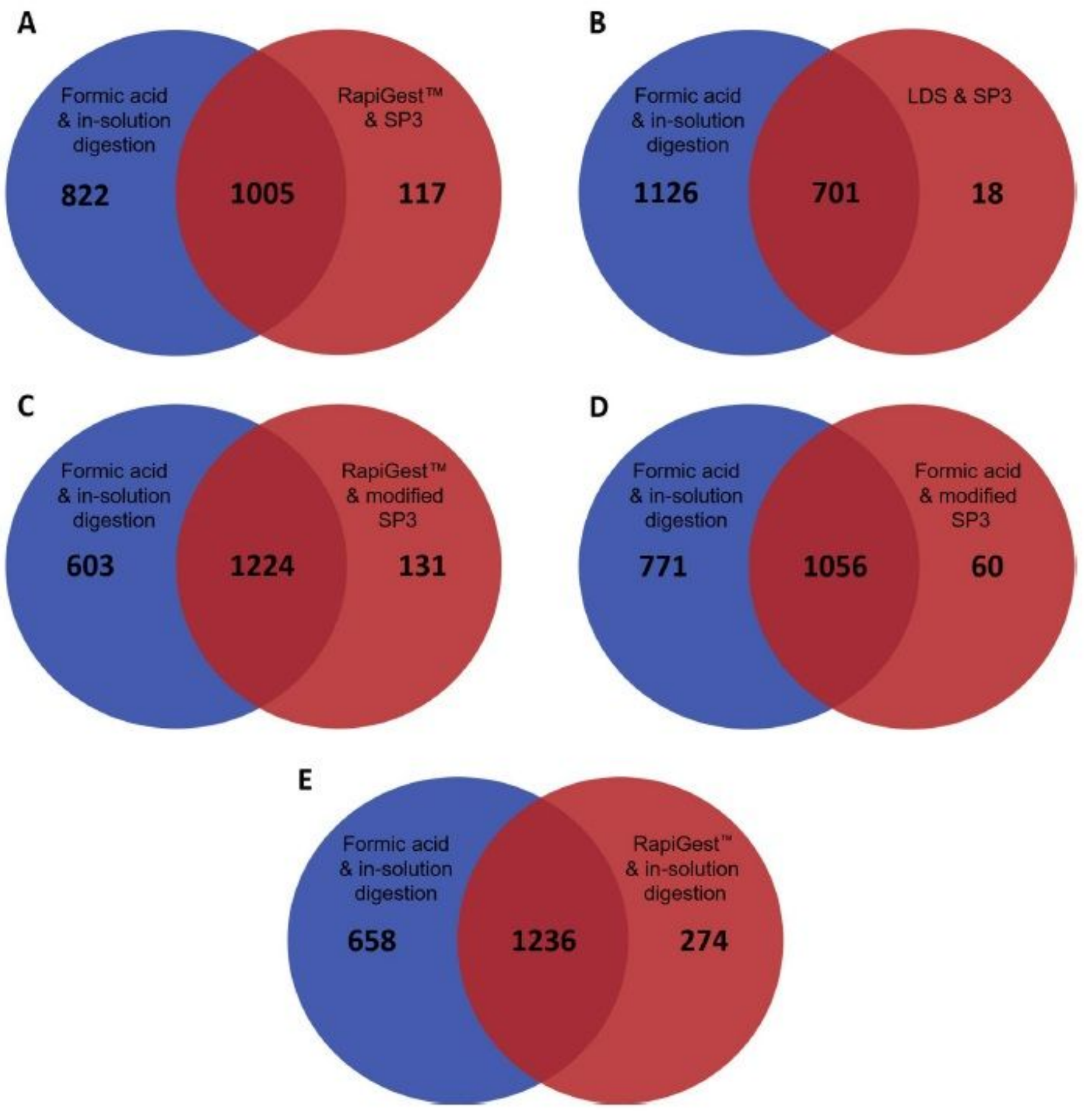

\section{Figure 3}

Venn-Diagrams of identified Protein Groups between the different protocols. For each protocol, all Protein Groups were used that were identified in all three replicates. Based on the UniProt accession number the list of identified Protein Groups from formic acid \& in-solution digestion was compared to all other protocols (A: RapiGest ${ }^{\text {TM }}$ \& SP3; B: LDS \& SP3; C: RapiGest ${ }^{\text {TM }}$ \& modified SP3; D: formic acid \& modified SP3; E: RapiGest ${ }^{\mathrm{TM}} \&$ in-solution digestion). 


\section{Supplementary Files}

This is a list of supplementary files associated with this preprint. Click to download.

- SupplementaryMaterial.docx 